\title{
Giant Aortic Arch Aneurysm and Cardio-vocal Syndrome: Still An Open-surgery Indication
}

\author{
Jose M. Garrido a, d, Maria Esteban ${ }^{\mathrm{b}}$, Juan Larab ${ }^{\mathrm{b}}$ Jose F. Rodriguez-Vazquez ${ }^{\mathrm{c}}$, \\ Samuel Verdugo-Lopez ${ }^{c}$, Salvador Lopez-Checa ${ }^{\mathrm{b}}$
}

\begin{abstract}
The Cardio-vocal Syndrome (Ortner's syndrome) is described as hoarseness due to the left recurrent laryngeal nerve palsy, caused by a specific cardiovascular pathology. In this case, we present a patient with a giant aortic arch aneurysm with an initial clinical presentation of Cardio-vocal Syndrome. The conventional opensurgery, instead of endovascular approach, was useful to control the morbidity from the compressive effect of adjacent structures, also preventing the aortic rupture. We strongly recommend analyzing carefully the individual case and the clinical targets to resolve, because the new technologies are not always the most effective therapeutic response.
\end{abstract}

Keywords: Aortic aneurysm; Cardio-vocal syndrome; Ortner's Syndrome

\section{Introduction}

The Cardio-vocal syndrome (Ortner's syndrome) [1] is produced by the palsy of the left recurrent laryngeal nerve due to different cardiovascular diseases. It was initially associated

Manuscript accepted for publication October 14, 2011

${ }^{a}$ Department of Cardiac Surgery, Department of Anatomy and Embryology II, Ramon y Cajal Hospital, Complutense University, Madrid, Spain

${ }^{\mathrm{b}}$ Department of Cardiovascular Surgery, Virgen de las Nieves Hospital, Granada, Spain

${ }^{\mathrm{c}}$ Department of Anatomy and Embryology II, Complutense Unversity,

Mardid, Spain

${ }^{\mathrm{d} C o r r e s p o n d i n g ~ a u t o r: ~ J o s e ~ M . ~ G a r r i d o, ~}$

Email: josemanuel.garrido@med.ucm.es

doi:10.4021/cr101w with left atrium enlargement and mitral valve pathology. In this sense, vocal cord paralysis is caused by a compression of the left recurrent laryngeal nerve between the pulmonary artery (enlarge and hypertensive in end-stage mitral valve disease), the aorta, and the ligamentum arteriosum. However other causes have been recently described such as penetrating atherosclerotic ulcers of the thoracic aorta [2], atrial mixoma, mitral regurgitation, congenital heart disease or, rarely, aortic aneurysms.

In this work, we present a patient with a giant aortic arch

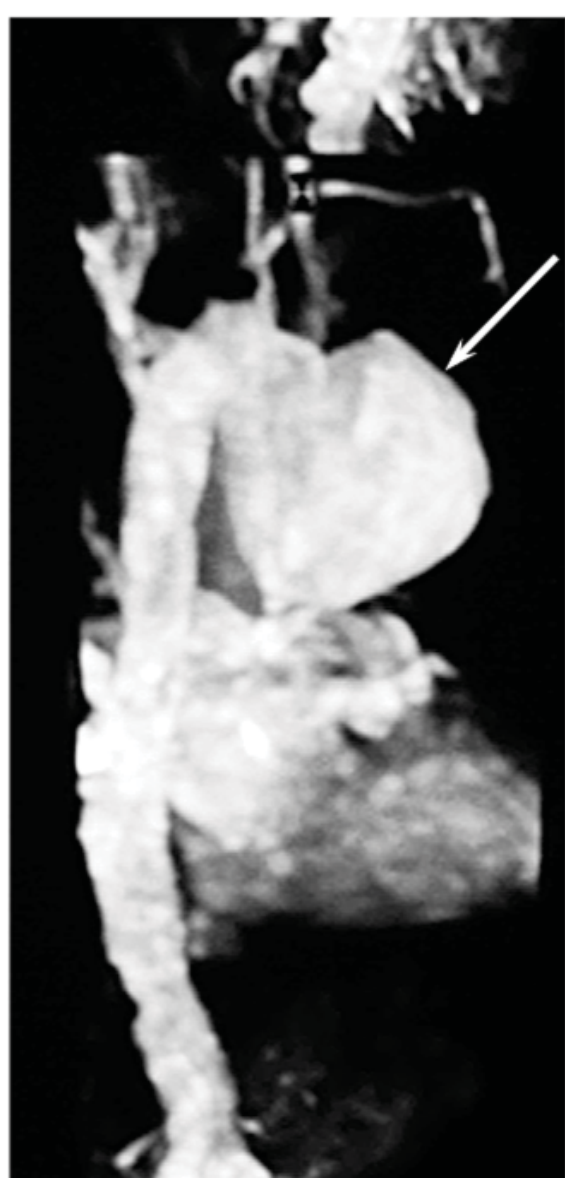

Figure 1. Giant saccular aortic arch aneurysm (white arrow) diagnosed by magnetic resonance imaging (MRI). 


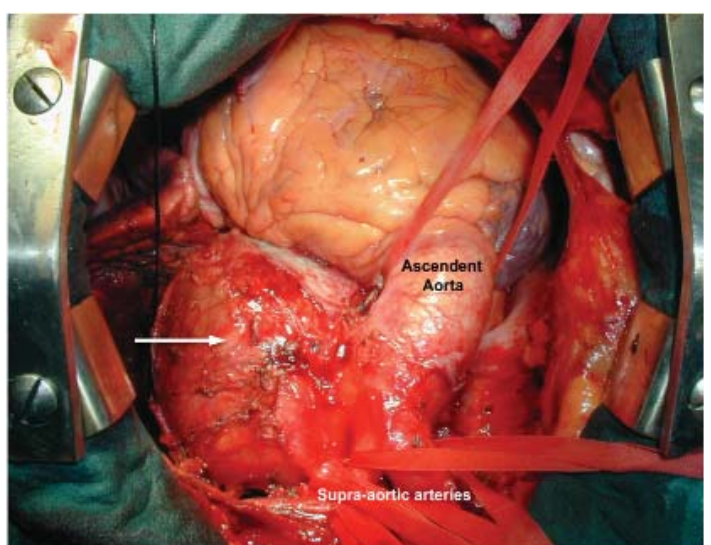

Figure 2. Intra-operative view of a giant aortic arch aneurysm (white arrow).

aneurysm diagnosed from a cardio-vocal syndrome presentation. A saccular aneurysm of the aortic arch is a very rare cause of hoarseness. This symptom, isolated or together with the presence of dysphagia, dyspnea or chest pain, constitutes a clear indication for surgical treatment of patients with aortic arch aneurysms [3].

\section{Case Report}

We present a 75 years old man, ex-smoker, with a history of arterial hypertension, chronic obstructive pulmonary disease and chronic renal failure. Since the past month, the patient has clinical symptoms of hoarseness and progressive dysphagia. This situation has advanced to dyspnea and thoracic pain in the last week.

Using magnetic resonance imaging (Fig. 1), we diagnosed a giant saccular aneurysm involving the basal portion of the aortic arch, at the opposite side of the origin of supraaortic arteries, with a significant mural thrombus, compressing the trachea, the left recurrent laryngeal nerve at the aortopulmonary window and the oesophagus [4]. The dimensions were $15 \mathrm{~cm}$ of external diameter and $8 \mathrm{~cm}$ of internal diameter (Fig. 2).

In this case, we decided to treat the aortic pathology by open surgery instead of using a hybrid procedure [5] that comprises the endovascular graft exclusion of the aortic arch and the translocation of the supra-aortic arteries- due to the predominance of compressive symptoms and the important mural thrombus (Fig. 3). Thus, the patient was operated using partial cardiopulmonary byspass, without cardiac arrest, clamping the aneurismal neck. We proceeded to open and resect the aneurysm. Afterward we reconstructed the aortic wall with a Dacron patch and we reinforce the aortic wall with fibrin sealant (Fig. 4).

The patient course was favourable, improving his respiratory function, disappearing the dyspnea and dysphagia,

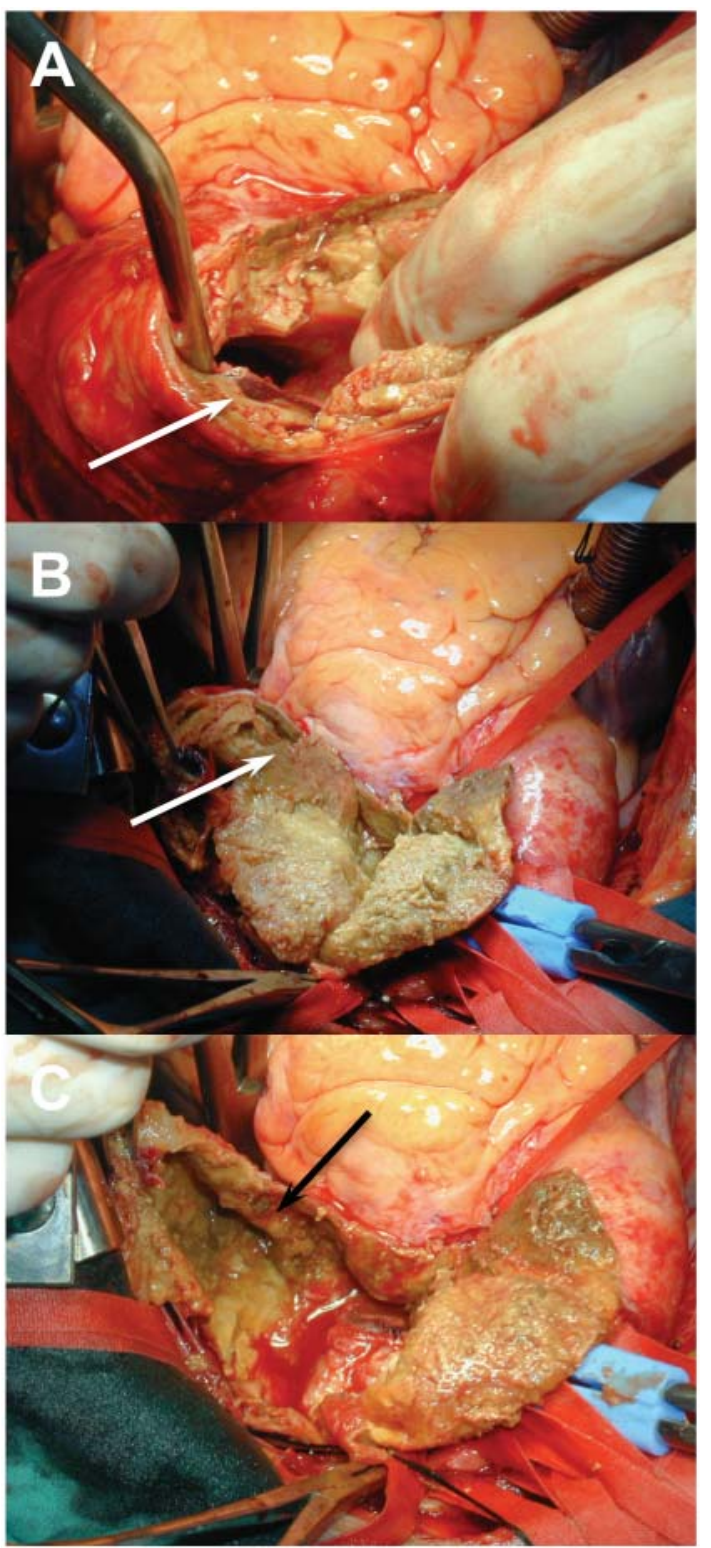

Figure 3. Important mural thrombus [3A and 3B (white arrow]. Thinned aneurysmal wall after thrombus removing [3C (black arrow)].

and reducing the symptoms of hoarseness.

\section{Discussion}

Hoarseness of voice, caused by a paralysis of the left recurrent laryngeal nerve due to left atrium enlargement in mitral valve stenosis, was described by Ortner in 1987. However, this syndrome, actually called Cardio-vocal syndrome, was associated with other specific cardiovascular pathologies, including severe pulmonary hypertension, congenital diseases, mitral valve disorders, iatrogenic causes or aortic aneurysm [4]. 


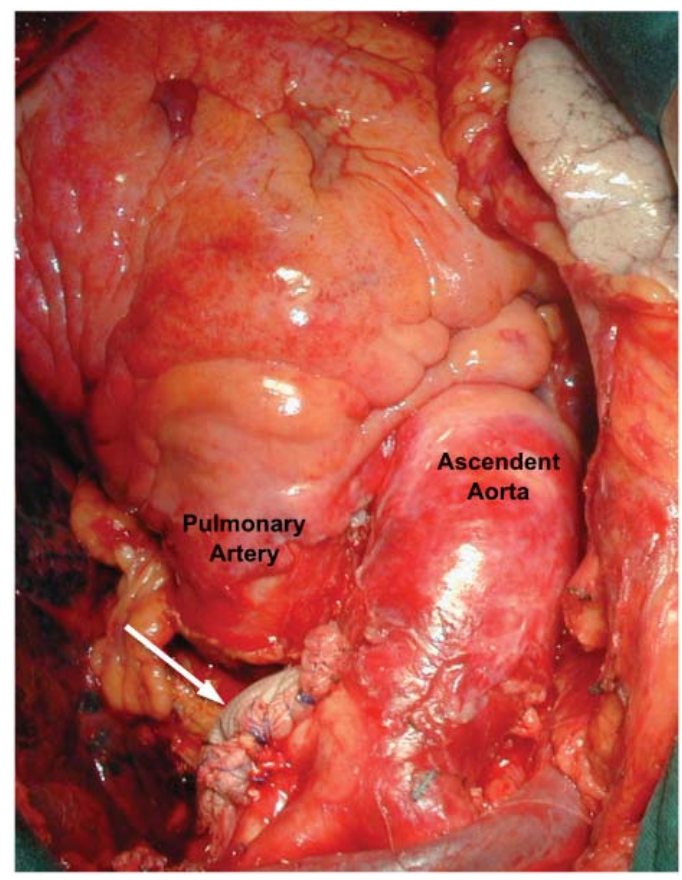

Figure 4. The aortic wall repair was made using a Dacron patch (white arrow) after the complete resection of the aneurysm.

Hoarseness, in a cardio-vocal syndrome context, together with the presence of dysphagia, dyspnea and chest pain, constitutes a clear indication for the surgical treatment of aortic aneurysms [3]. The interventional technologies using hybrid procedures has decreased the mortality and morbidity for surgery of aortic arch aneurysms. We can distinguish two different types of hybrid procedures depending on the aortic arch approach [5]. Type I procedures, including the "frozen elephant trunk" are specially indicated in a concomitant aneurysms of aortic arch and descending aorta [6]. For its part, type II procedures consider the endovascular repair concept as the main technique, excluding the aortic arch with the stent graft. The open surgical step is limited to revascularize the supra-aortic arteries. Type II hybrid procedures is useful to treat lesions limited to the aortic arch in which the stent graft may exclude the arch successfully. Thus, in our case we could treat the aortic aneurysm using a type II hybrid procedure, preventing the aortic rupture with less risk for the patient. Nevertheless, probably we would not resolve the significant compressive symptoms with the isolated endovascular exclusion of the aneurysm, due to the giant size and the important mural thrombus.

In this case we decided to schedule the patient for opensurgery as the most appropriate surgical technique. With this approach we eliminated the morbidity from the compressive effect of adjacent structures, also preventing the aortic rupture. On the contrary, with the exclusion of the giant aneurysm by a stent graft we could not ensure the control of the compressive symptoms such as dysphagia, dyspnea or hoarseness.

We strongly recommend analyzing carefully the individual case and the clinical targets to resolve. In this sense, the new technologies are not always the most effective therapeutic response [7].

\section{Conflicts of Interest}

There is no conflict of interest of any of the authors.

\section{References}

1. Fennessy BG, Sheahan P, McShane D. Cardiovascular hoarseness: an unusual presentation to otolaryngologists. J Laryngol Otol. 2008;122(3):327-328.

2. Mickus TJ, Mueller J, Williams R. An uncommon cause of Ortner syndrome. J Thorac Imaging. 2010;25(3):W8284.

3. Hiratzka LF, Bakris GL, Beckman JA, Bersin RM, Carr VF, Casey DE, Jr., Eagle KA, et al. 2010 ACCF/ AHA/AATS/ACR/ASA/SCA/SCAI/SIR/STS/SVM guidelines for the diagnosis and management of patients with Thoracic Aortic Disease: a report of the American College of Cardiology Foundation/American Heart Association Task Force on Practice Guidelines, American Association for Thoracic Surgery, American College of Radiology, American Stroke Association, Society of Cardiovascular Anesthesiologists, Society for Cardiovascular Angiography and Interventions, Society of Interventional Radiology, Society of Thoracic Surgeons, and Society for Vascular Medicine. Circulation. 2010;121(13):E266-369.

4. Plastiras SC, Pamboucas C, Zafiriou T, Lazaris N, Toumanidis S. Ortner's syndrome: a multifactorial cardiovocal syndrome. Clin Cardiol. 2010;33(6):E99-100.

5. Koullias GJ, Wheatley GH, 3rd. State-of-the-art of hybrid procedures for the aortic arch: a meta-analysis. Ann Thorac Surg. 2010;90(2):689-697.

6. Garrido JM, Miguelena J, Munoz R, Prada P, Epeldegui A. Repair of multiple aneurysms of the thoracic aorta with a hybrid prosthesis. J Card Surg. 2011;26(2):197200.

7. Dronavalli VB, Loubani M, Riley P, Bonser RS. Failure to exclude a saccular arch aneurysm during hybrid repair: arch replacement without cerebral circulatory arrest. Interact Cardiovasc Thorac Surg. 2009;9(4):677679. 